\title{
Daylength influences pelage and plasma prolactin concentrations but not reproduction in the prairie vole, Microtus ochrogaster
}

\author{
Laura Smale* ${ }^{*}$, R. J. Nelson $\ddagger$ and I. Zucker $\dagger$ \\ †Department of Psychology, University of California, Berkeley, CA 94720, U.S.A. and \\ $\ddagger$ Departments of Psychology and Population Dynamics, The Johns Hopkins University, Baltimore, \\ $M D 21218$, U.S.A.
}

\begin{abstract}
Summary. Short daylengths did not affect testes weight or spermatogenic index in male voles or uterine weight in female voles. Short daylengths did stimulate the growth of a winter pelage in both sexes; short-day voles had longer underhairs and guard hairs and a thicker, more dense pelage than did long-day voles. Plasma prolactin concentrations were five times higher in long-day than in short-day females and $25 \%$ higher in longday males than in short-day males. The effect of short daylength on pelage was prevented by pinealectomy. We suggest that the growth of a winter coat is an obligate adaptation for winter survival, stimulated by exposure to short daylengths, but that changes in breeding activity are facultative and dependent to a greater extent on other cues for seasonal synchronization.
\end{abstract}

Keywords: vole; photoperiodism; prolactin; pelage; reproduction

\section{Introduction}

The prairie vole, Microtus ochrogaster, a small monogamous microtine rodent found throughout much of the midwestern United States, typically experiences long cold winters and hot summers. Adjustments that enhance winter survival include a reduction in body weight, a reduced metabolic rate and the development of a winter pelage (Cherry \& Verner, 1975).

Prairie voles are perhaps best described as facultative seasonal breeders (Negus \& Berger, 1972); reproductive activity is reduced in the winter of some but not all years (Rose \& Gaines, 1976; Getz et al., 1979). Non-reproductive adaptations, however, may be of a more obligate nature. All voles could presumably benefit every winter from the increased insulation provided by a thicker pelage. If this reasoning is valid, then proximate cues for phasing reproductive and non-reproductive adaptations should differ. In particular, daylength, the most reliable predicator of phase within the annual geophysical cycle, might be used to regulate non-reproductive but not reproductive seasonal adjustments. One aim of the present study was to test this prediction.

Short daylengths inhibited reproductive development in $75 \%$ of male prairie voles born in the laboratory to wild-caught individuals (Nelson, 1985a). In subsequent studies of voles maintained in the laboratory for 4 and 5 generations, daylength did not affect testicular growth (unpublished observations). Among adult females, photoperiod influenced neither pregnancy nor lactation (Nelson, 1985b). Rates of reproductive development have yet to be assessed in prepubertal female prairie voles raised in different photoperiod regimens.

\footnotetext{
*Present address: Department of Psychiatry and Behavioral Science, Health Sciences Center, State University of New York, Stony Brook, NY 11794, U.S.A.
} 
In this study we examined reproductive and non-reproductive responses to daylength in young male and female prairie voles from a colony that had been maintained in the laboratory for 4 to 5 generations.

\section{Materials and Methods}

\section{Animals and methods}

Animals. All voles were derived from a laboratory colony established with 12 pairs of adult prairie voles trapped in south central Illinois in May 1983. Breeding pairs were constituted randomly except that brother-sister matings were avoided. Breeding pairs were maintained at $22 \pm 2^{\circ} \mathrm{C}$ in a $16 \mathrm{~h}$ light: $8 \mathrm{~h}$ dark photoperiod with food and water provided ad libitum. Voles were weaned and housed individually at 21 days of age.

Pelage. Fur depth and under hair and guard hair length were measured with calipers to the nearest $0.1 \mathrm{~mm}$. Under hairs were distinguished from guard hairs on the basis of thickness and colour. Three guard hairs and one under hair were measured from each animal. Under hairs were measured directly from the animals' coat but guard hairs were removed and then measured. Pelage density was measured by shaving and weighing $( \pm 0.01 \mathrm{mg})$ a $1 \mathrm{~cm}^{2}$ patch of fur from the posterior dorsal surface. This was accomplished by placing the vole in a plastic 'girdle' with a $1 \times 1 \mathrm{~cm}$ square hole and shaving all of the hair within the boundaries of that hole. All measures of pelage development were done by an investigator blind to the animals' experimental condition.

Histology. Testes were stored in buffered formalin and then dehydrated, embedded in paraffin wax, sectioned at $5 \mu \mathrm{m}$ and stained with haematoxylin and eosin. Testicular tissue was examined microscopically and rated for spermatogenesis according to the rating system described by Grocock \& Clarke (1974). In this classification, a value of $5=$ large seminiferous tubules with complete spermatogenesis; $4=$ complete spermatogenesis with a decreased number of spermatozoa and elongated spermatids; $3=$ fewer spermatozoa, but elongated spermatids present; 2 = elongated spermatids absent; 1 = only Sertoli cells, spermatogonia and primary spermatocytes present; and $0=$ only Sertoli cells and spermatogonia present.

Radioimmunoassays. Prolactin was measured in duplicate 10- $\mu$ l samples of plasma with an RIA developed using a rabbit antibody to deer mouse prolactin (Peromyscus maniculatus bairdii; Marr et al., 1983). Assay reagents and blood plasma were diluted in $0.1 \%$ gel phosphate-buffered saline. A double-antibody RIA was adapted to measure prolactin in small samples of vole blood plasma (Desjardins \& Lopez, 1983). ${ }^{125}$ I-labelled deer mouse prolactin was prepared and purified on Sephadex G-75 3 days before the assay.

Four standard curves were produced with 12 dose levels ranging from 0.02 to 4.0 ng prolactin/assay tube. Final hormone concentrations were expressed in terms of nanogram equivalents of a highly purified deer mouse prolactin per millilitre of prairie vole blood plasma. Reference and unknown sample values were based on a weighted non-linear least-squares regression of the standard curves (Ellis \& Desjardins, 1982). All plasma samples were measured in the same assay. The intra-assay coefficients of variation for pools of plasma obtained from intact or castrated males or intact female voles were $4.9 \%, 5.5 \%$ and $4.0 \%$, respectively. Plasma from normal or castrated voles inhibited the assay between 20 and $85 \%$. The minimum amount of prolactin detectable in unknown samples was $0 \cdot 25 \mathrm{ng} / 10 \mu \mathrm{l}$ plasma.

Inhibition curves observed with plasma obtained from normal or gonadectomized male and female voles were parallel to the inhibition curve obtained with the reference preparation of prolactin. Furthermore, the addition of increasing amounts of vole plasma in a fixed volume $(10 \mu \mathrm{l})$ of blood plasma taken from a normal male prairie vole produced a dose-dependent increment in immunoreactive prolactin. Taken together, these results indicate that the deer mouse prolactin assay system detects immunoreactive prolactin in prairie vole plasma samples with precision and sensitivity.

Statistics. In Exp. 1, comparisons between males were analysed with independent 2-tailed $t$ tests and comparisons amongst female treatment groups with ANOVA. A $\chi^{2}$ test was used for the analysis of treatment effects on oestrous behaviour and vaginal patency. Data from Exp. 2 were analysed with ANOVA. Effects were considered significant if $P<0.05$.

\section{Experiment 1}

Adult voles were paired and maintained in long days ( $16 \mathrm{~L}: 8 \mathrm{D})$ on a diet of Purina mouse chow and Purina guineapig chow until young were born. Beginning at parturition, all animals were fed a special diet (Custom mouse mix, Brookfield Products, Murray, UT, U.S.A.) free of the plant stimulator compound 6-methoxybenzoaxazolinone. At this time, half of the litters $(n=10)$ were transferred to short days (8L:16D) where they remained for the duration of the experiment.

Females. Female prairie voles are dependent upon exposure to males for the induction of oestrus and remain in anoestrus indefinitely when housed alone (Richmond \& Conaway, 1969). To determine whether photoperiod influences the process of induction of oestrus, half of the females from each photoperiod condition were exposed to 
adult males at 45 days of age. Male-exposed females were housed with 1 fecund adult long-day male for $56 \mathrm{~h}$; control females were left alone during this interval. All females were then weighed, examined for vaginal patency and tested with an unfamiliar stud male for $5 \mathrm{~min}$ for evidence of behavioural oestrus.

Females were decapitated $24 \mathrm{~h}$ later, trunk blood was collected, and uteri dissected and weighed. Ovaries were inspected for evidence of corpora lutea. Fur length, depth and density were recorded. Blood was centrifuged and stored frozen until assayed for prolactin.

Males. At 45 days of age, males were weighed and decapitated. Trunk blood was collected and centrifuged immediately and plasma was stored frozen until assayed for prolactin. Measures for fur development were obtained as described above. Testes were dissected, weighed and prepared for histological examination.

\section{Experiment 2}

Adult male voles ( $>90$ days) born into and maintained from birth in long days (16L:8D) were housed individually and provided with water and food (Purina Mouse Chow and Purina guinea-pig chow) ad libitum.

All voles were pinealectomized or sham-pinealectomized under ketamine anaesthesia. A small $(2 \mathrm{~mm})$ circular piece of skull centred around lambda was cut out with a trephine attached to a hand-held drill. For sham operations there was no further intervention. For pinealectomies, the pineal gland was excised from the venous sinus with forceps and placed on a glass slide. Tissue was inspected to verify that it included the pineal gland. The skull plate was replaced, cotton gauze applied to retard bleeding, and the skin clamped with wound clips.

After a recovery period of at least 2 weeks, half of the voles from each group were transferred to the short-day photoperiod (6L:18D) and the remaining animals were kept in the original long-day (LD) photoperiod until the experiment was ended 10 weeks later. At this time all voles were given a lethal dosage of pentobarbitone sodium and weighed; testes were dissected, weighed and pelage characteristics measured, as described above. Testes were stored in formalin until they were dehydrated, embedded in paraffix wax, sliced at $6 \mu \mathrm{m}$ and stained with haematoxylin and eosin. Spermatogenic development was rated as described above.

\section{Results}

\section{Experiment 1}

\section{Females}

Reproduction. Uterine weight was not affected by photoperiod but was increased by exposure to males $(P<0.001$; Table 1). A female was judged in oestrus if she mated, or a vaginal plug was visible after cohabitation with a male, or if her ovaries had corpora lutea (a reliable index of mating in this reflex-ovulating species). None of the control females exhibited behavioural oestrus. Photoperiod did not affect the probability that exposure to a male would induce oestrus: $7 / 10$ females from $6 \mathrm{~L}: 18 \mathrm{D}$ were in oestrus after $56 \mathrm{~h}$ of cohabitation with a male, compared to $4 / 8$ females from 16L:8D. Vaginal patency was unaffected by photoperiod or exposure to males.

Pelage. All 4 measures of pelage development were affected by photoperiod and none was influenced by exposure to a male. Therefore, data from the two short-day groups were combined

Table 1. Reproductive indices and prolactin concentrations in voles in Exp. 1

\begin{tabular}{lccr}
\hline & $\begin{array}{c}\text { No. of } \\
\text { voles }\end{array}$ & $\begin{array}{c}\text { Uterine/testes } \\
\text { weight }(\mathrm{mg})\end{array}$ & \multicolumn{1}{c}{$\begin{array}{c}\text { Prolactin } \\
(\mathrm{ng} / \mathrm{ml})\end{array}$} \\
\hline Females & & & \\
$\quad$ Long-day controls & 8 & $17 \cdot 8 \pm 1 \cdot 2$ & $24 \cdot 6 \pm 5 \cdot 5$ \\
$\quad$ Long-day male-exposed & 8 & $39 \cdot 4 \pm 6 \cdot 8$ & $12 \cdot 7 \pm 3 \cdot 0$ \\
$\quad \begin{array}{l}\text { Short-day controls } \\
\quad \text { Short-day male-exposed }\end{array}$ & 10 & $20 \cdot 7 \pm 2 \cdot 7$ & $5 \cdot 9 \pm 2 \cdot 5$ \\
$\quad 10$ & $39 \cdot 3 \pm 4 \cdot 0$ & $10 \cdot 9 \pm 3 \cdot 4$ \\
$\quad \begin{array}{l}\text { Lales } \\
\quad \text { Song-day }\end{array}$ & 12 & $367 \cdot 3 \pm 34 \cdot 4$ & $22 \cdot 6 \pm 1 \cdot 4$ \\
$\quad$ & 19 & $328 \cdot 4 \pm 29 \cdot 2$ & $16 \cdot 2 \pm 2 \cdot 3$ \\
\hline
\end{tabular}

Values are mean \pm s.e.m. 

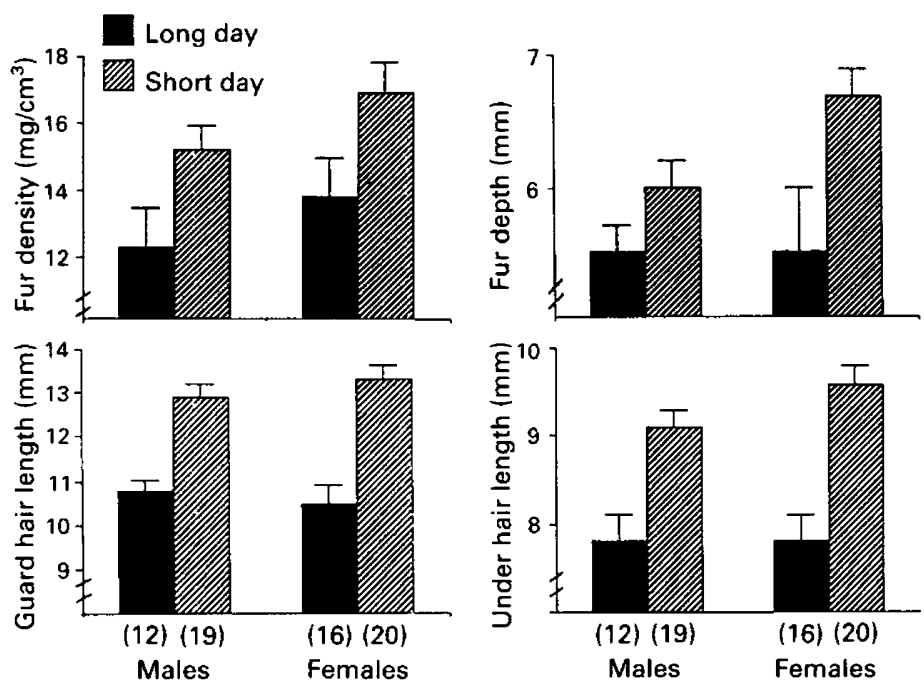

Fig. 1. Pelage measures of male and female voles in Exp. 1. Values are mean \pm s.e.m. for the no. of animals indicated in parentheses, respectively. Differences between short- and long-day voles were significant $(P<0.05)$ for all measures with the exception of fur depth among the males $(P<0 \cdot 07)$.

and compared with data from the two long-day groups (Fig. 1). Short daylengths increased fur depth $(P<0.001)$, under hair length $(P<0.001)$, guard hair length $(P<0.001)$ and fur density $(P<0.02)$.

Prolactin. Prolactin concentrations were influenced by photoperiod $(P<0.01)$ but not by exposure to a male (Table 1). In addition, the interaction between photoperiod and male exposure was significant $(P<0.05)$. Prolactin concentrations were nearly 5 -fold greater in long-day than short-day controls. Exposure to males did not influence prolactin values in short-day females and decreased concentrations in long-day females, but the latter trend was not statistically significant.

Body weight. Body weight was influenced by photoperiod $(P<0.005)$ but not by exposure to a male. Long-day females weighed substantially less than short day females $(25.2 \pm 1.3$ vs $32 \cdot 2 \pm 1 \cdot 6$ g respectively).

\section{Males}

Reproduction. Absolute testicular weight was not affected by photoperiod $(P>0.05$; Table 1$)$ but relative weight of testes was greater in long-day than short-day males $(P<0.006)$. A significant correlation between testicular and body weights was evident within each treatment group $(r=0.74$, $P<0.001$ among short-day males, and $r=0.72, P<0.05$ among long-day males). The spermatogenic index did not differ between the groups $(4 \cdot 24 \pm 0.16$ and $4.18 \pm 0.11$ for long- and short-day voles respectively) and mature spermatozoa were present in testes of all voles.

Pelage. Three of the 4 fur development measures were significantly influenced by photoperiod (Fig. 1). Under hairs and guard hairs were longer $(P<0.001)$ and fur was more dense $(P<0.03)$ in short-day than long-day voles. Fur depth was greater in short-day than long-day voles, but this difference was not significant $(P<0 \cdot 07)$.

Prolactin. Prolactin concentrations were higher in long-day than short-day males $(22.6 \pm 1.4 \mathrm{vs}$ $16.3 \pm 2.3 \mathrm{ng} / \mathrm{ml} ; P<0 \cdot 05)$.

Body weight. Males kept in long days were significantly lighter than those kept in short days $(24 \cdot 7 \pm 1 \cdot 2$ vs $29 \cdot 7 \pm 1 \cdot 4 \mathrm{~g}$ respectively; $P<0 \cdot 02)$ 

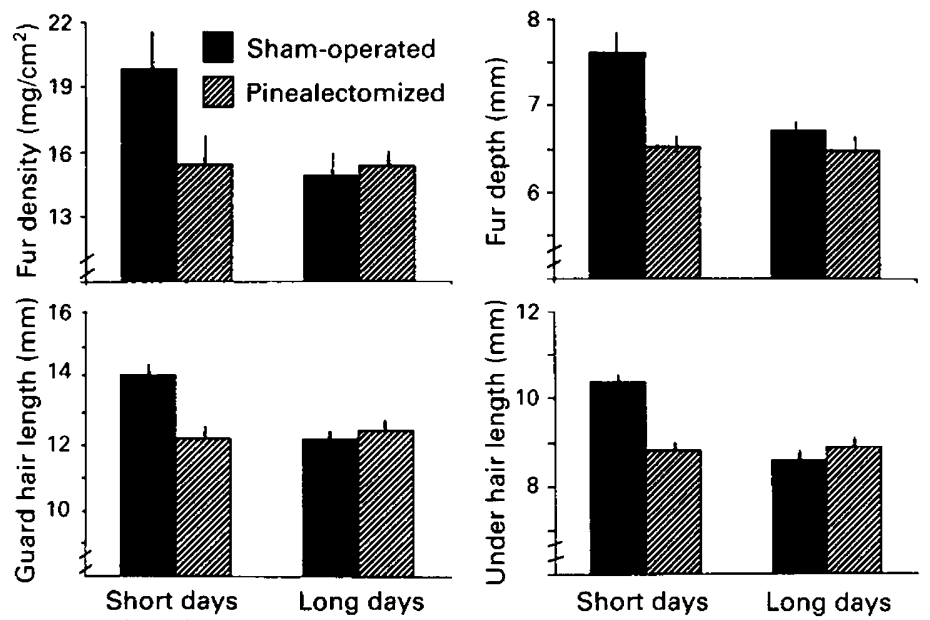

(14) (14)

(12) (13)

(14) (14)

(12) (13)

Fig. 2. Pelage measurements of adult male voles sham-pinealectomized or pinealectomized and kept in short or long days for 10 weeks. Values are mean \pm s.e.m., for the sample sizes given in parentheses.

Table 2. Testicular indices of voles from Exp. 2

\begin{tabular}{lccc}
\hline & $\begin{array}{c}\text { No. of } \\
\text { voles }\end{array}$ & $\begin{array}{c}\text { Testes weight } \\
(\mathrm{mg})\end{array}$ & $\begin{array}{c}\text { Spermatogenic } \\
\text { index }\end{array}$ \\
\hline $\begin{array}{c}\text { Long-day } \\
\text { sham-operated }\end{array}$ & 12 & $661 \cdot 7 \pm 65 \cdot 4$ & $4 \cdot 32 \pm 0 \cdot 17$ \\
$\begin{array}{c}\text { Long-day } \\
\text { pinealectomized }\end{array}$ & 13 & $741 \cdot 2 \pm 79 \cdot 7$ & $4 \cdot 17 \pm 0 \cdot 13$ \\
$\begin{array}{c}\text { Short-day } \\
\text { sham-operated }\end{array}$ & 14 & $535 \cdot 4 \pm 91 \cdot 3$ & $4 \cdot 08 \pm 0 \cdot 20$ \\
$\begin{array}{c}\text { Short-day } \\
\text { pinealectomized }\end{array}$ & 14 & $762 \cdot 7 \pm 63 \cdot 4$ & $4 \cdot 39 \pm 0 \cdot 13$ \\
\hline
\end{tabular}

Values are mean \pm s.e.m.

\section{Experiment 2}

Pelage. Depth, under hair length and guard hair length were all affected by photoperiod and by pinealectomy (Fig. 2). Short photoperiods increased the dimensions of these 3 pelage characteristics, but only in sham-pinealectomized voles; pinealectomy prevented these responses to short days. Fur density was increased by short daylengths but was not significantly influenced by pinealectomy $(P=0 \cdot 13)$.

Testes. Testicular weight and spermatogenic index were unaffected by photoperiod or pinealectomy (Table 2).

\section{Discussion}

Photoperiod did not affect reproduction but did influence the growth of a winter pelage in prairie voles. The effect of photoperiod on fur was blocked by removal of the pineal gland and may be mediated by changes in prolactin concentrations. 
None of the measures of female reproduction monitored in this experiment was influenced by daylength. Exposure to males stimulated reproductive function in females reared in long and short daylengths. Uterine weight was nearly doubled in females exposed to males for $56 \mathrm{~h}$ compared to controls. Evidence of behavioural oestrus was obtained in $70 \%$ of short-day females and in $50 \%$ of long-day females exposed to males.

Daylength does not appear to influence the neuroendocrine pathway through which male pheromones induce oestrus. If photoperiod is to affect reproductive activity in female prairie voles, it must do so through some post-copulatory process essential for ovum implantation or maintenance of pregnancy. It also remains possible that ovulation rate is influenced by daylength.

Photoperiod exerted little or no effect on gonadal development of male prairie voles or on the maintenance of testicular function in adults. In Exp. 1, absolute testes weight was unaffected by daylength, but relative testes weight was greater in long-day than short-day males. The latter effect is attributable to reduced body weight of long-day males. Histological analysis confirmed that short daylengths did not inhibit testicular development. The spermatogenic index was unaffected by photoperiod and spermatozoa were present in the testes of all males in each photoperiod condition in both experiments.

The failure to observe an effect of daylength on testicular development contrasts with an earlier report (Nelson, 1984). The voles studied by Nelson (1984) were offspring of wild-caught animals, while voles used in the present study were 4 and 5 generations removed from the wild stock. Even among offspring of wild-caught voles, however, $25 \%$ underwent rapid reproductive growth in spite of exposure to short daylengths (Nelson, 1985a). The reproductive rate of this subpopulation may have exceeded that of voles responsive to short daylengths and over 4 generations of random breeding in the laboratory, genotypes conferring responsiveness of the reproductive apparatus to photoperiod may have been eliminated (Clarke, 1977; Nelson, 1985b; Spears \& Clarke, 1987).

In contrast to its lack of effect on reproductive processes, photoperiod markedly affected every measure of pelage growth in female prairie voles. In short days, females developed a deeper, more dense and longer coat of fur than in long days. Among males, 3 of the 4 measures of pelage were substantially influenced by photoperiod in Exp. 1 and all 4 measures were increased by exposure to short days in Exp. 2. These results are consistent with findings from other microtine rodents (Al Khateeb \& Johnson, 1971; Dark \& Zucker, 1983).

The effect of daylength on pelage may be mediated through changes in prolactin secretion. Plasma prolactin concentrations were substantially reduced in males and females maintained in short daylengths (Exp. 1). This effect was most pronounced in females; prolactin values were 5 times higher in control long-day than short-day females.

Evidence from several other mammals indicates that short daylengths stimulate the development of a winter pelage by inhibiting prolactin secretion (e.g. Siberian hamsters: Duncan \& Goldman, 1983; mink: Martinet et al., 1984). In these species, the development of a winter coat in short daylengths coincides with a decrease in circulating prolactin and is blocked by daily injections of prolactin. Stimulatory effects of short-days on fur growth also are prevented by prolactin injections in the meadow vole, M. pennsylvanicus (L. Smale, T. Lee \& I. Zucker, unpublished observations).

The pineal gland appears to mediate photoperiodic effects on pelage growth in prairie voles. Pinealectomy eliminated the effect of photoperiod on pelage depth and under hair and guard hair length but only marginally influenced fur density. Pinealectomy had no effect on testes size or spermatogenesis. These findings are consistent with results from several mammalian species, including the closely related vole, M. agrestis (Versi et al., 1983).

It is likely that in voles, as in other rodents (Goldman \& Darrow, 1983), the period of elevated nocturnal melatonin secretion is extended in short days and that this, in turn, triggers the effects of daylength on various photoperiodic traits. The present results suggest that the dissociation of reproductive and non-reproductive responses is a post-pineal phenomenon. Factors such as genotype, age, diet or temperature may alter the sensitivity of the pituitary-gonadal axis to melatonin 
and thus to photoperiod. In contrast, prolactin secretion may always decrease in response to melatonin and fur growth may therefore respond to photoperiod under a wider range of conditions.

We suggest that prior designations of animals as photoperiodic or non-photoperiodic are incorrect (Johnston \& Zucker, 1980; Beasley et al., 1981). It is more appropriate to consider individual traits as subject to photoperiodic regulation. This is not a trivial distinction since the mechanisms for photoperiodic time measurement must be in place even if only a single characteristic responds to daylength.

Photoperiodic regulation of reproduction appears to be a highly labile phenomenon rapidly eliminated by random breeding in the laboratory or by increased food availability in the field (Cole \& Batzli, 1979). This lability may be important to voles because winter reproduction may be highly advantageous during winters when high quality food is available or the temperature is unseasonably high. Seasonal reproduction in the field is variable in prairie voles (Keller \& Krebs, 1970). In contrast, a thicker coat of fur would presumably be advantageous every winter, regardless of local fluctuations in food supply or temperature (Smale, 1987). Decreasing daylength is a reliable predictor of the onset of generally lower temperatures, and thus a useful cue for the timing of the development of a winter pelage.

We thank Lisa Higa, Chris Tuthill and Claudine Dutaret for technical assistance. This research was supported by NIH grant HD 02982.

\section{References}

Al Khateeb, A. \& Johnson, E. (1971) Seasonal changes of pelage in the vole (Microtus agrestis) I. Correlation with changes in the endocrine glands. Gen. comp. Endocr. 16, 217-228.

Beasley, L.J., Johnston, P.G. \& Zucker, I. (1981) Photoperiodic regulation of reproduction in postpartum Peromyscus leucopus. Biol. Reprod. 24, 962-966.

Cherry, R.H. \& Verner, L. (1975) Seasonal acclimatization to temperature in the prairie vole (Microtus ochrogaster). Am. Mid. Nat. 94, 354-360.

Clarke, J.R. (1977) Long and short term changes in gonadal activity of field voles and bank voles. Oikos 29, 457-467.

Cole, R.F. \& Batzli, G.O. (1979) Nutrition and population dynamics of the prairie vole, Microtus ochrogaster, in central Illinois. J. Anim. Ecol. 48, 455-470.

Dark, J. \& Zucker, I. (1983) Short photoperiods reduce winter energy requirements in the meadow vole, Microtus pennsylvanicus. Physiol. Behav. 31, 699-702.

Desjardins, C. \& Lopez, M.J. (1983) Environmental cues evoke differential responses in pituitary-testicular function in deer mice. Endocrinology 112, 1398-1406.

Duncan, M.J. \& Goldman, B.D. (1983) Photoperiodic regulation of prolactin levels may mediate the annual pelage color cycle in the Djungarian hamster (Phodopus sungorus sungorus): mediation by melatonin. Endocrinology 113, 1268-1273.

Ellis, G.B. \& Desjardins, C. (1982) Male rats secrete luteinizing hormone and testosterone episodically. Endocrinology 110, 1618-1627.

Getz, L.L., Verner, L., Cole, F.R., Hofman, J.E. \& Avalos, D.E. (1979) Comparisons of population demography of Microtus ochrogaster and Microtus pennsylvanicus. Acta theriol. 24, 319-349.

Goldman, B.D. \& Darrow, J.M. (1983) The pineal gland and mammalian photoperiodism. Neuroendocrinology 37, 386-396.

Grocock, C.A. \& Clarke, J.R. (1974) Photoperiodic control of testes activity in the vole, Microtus agrestis. $J$. Reprod. Fert. 39, 337-347.

Johnston, P.G. \& Zucker, I. (1980) Photoperiodic regulation of the testes of adult white-footed mice (Peromyscus leucopus). Biol. Reprod. 23, 859-866.

Keller, B.L. \& Krebs, C.J. (1970) Microtus population biology. III. Reproductive changes in fluctuating populations of $M$. ochrogaster and $M$. pennsylvanicus in southern Indiana. Ecol. Monogr. 40, 263-294.

Marr, G.A., Colosi, P., Desjardins, C. \& Talamantes, F. (1983) Development and characterization of a homologous radioimmunoassay for deer mouse (Peromyscus maniculatus bairdii) prolactin. Life Sci. 33, 2305-2309.

Martinet, L., Allain, D. \& Weiner, C. (1984) Role of prolactin in the photoperiodic control of moulting in the mink (Mustela vison). J. Endocr. 103, 9-15.

Negus, N.C. \& Berger, P.J. (1972) Environmental factors and reproductive processes in mammalian populations. In Biology of Reproduction: Basic and Clinical Studies, pp. 89-98. Eds J. T. Velardo \& B. A. Kasprow. Bay Publisher, Mexico City.

Nelson, R.J. (1984) Proximate factors affecting reproduction in the prairie vole (Microtus ochrogaster ochrogaster). Ph.D. thesis, University of California, Berkeley.

Nelson, R.J. (1985a) Photoperiod influences reproduction in the prairie vole (Microtus ochrogaster). Biol. Reprod. 33, 596-602.

Nelson, R.J. (1985b) Photoperiodic regulation of reproductive development in male prairie voles: influence of laboratory breeding. Biol. Reprod. 33, 418-422. 
Richmond, M. \& Conaway, C.H. (1969) Induced ovulation and oestrus in Microtus ochrogaster. J. Reprod. Fert., Suppl. 6, 357-376.

Rose, R.K. \& Gaines, M.S. (1976) The reproductive cycle of Microtus ochrogaster in eastern Kansas. Ecol. Monogr. 48, 21-42.

Smale, L. (1987) Pheromones photoperiod and reproduction in the prairie vole (Microtus ochrogaster). Ph.D. thesis, University of California, Berkeley.
Spears, N. \& Clarke, J.R. (1987) Comparison of the gonadal response of wild and laboratory field voles (Microtus agrestis) to different photoperiods. J. Reprod. Fert. 79, 75-81.

Versi, E., Chiappa, S.A., Fink, G. \& Charlton, H.M. (1983) Pineal influences hypothalamic Gn-RH content in the vole, Microtus agrestis. J. Reprod. Fert. 67, 365-368.

Received 30 June 1987 